\title{
Treatment Options for Pediatric Diabetes
}

\author{
An Expert Interview with M Loredana Marcovecchio \\ Department of Paediatrics, University of Cambridge, Cambridge, UK
}

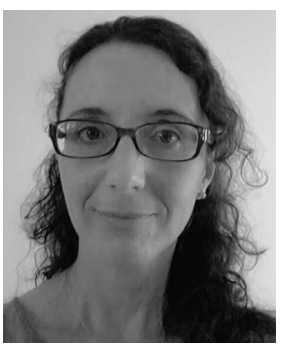

\section{Loredana Marcovecchio}

M Loredana Marcovecchio is a clinical researcher working in the field of pediatric diabetes at the Department of Paediatrics, University of Cambridge, UK. She graduated in Medicine at the University of Chieti (Italy) and obtained her PhD in physiopathology of metabolism and MD in pediatrics at the same university. Her main research interests are the early detection and prevention of vascular complications of childhood-onset diabetes, and immunotherapy for type 1 diabetes. Her work has led to a large number of publications in high impact journals and it has been recognized both nationally and internationally with several awards: L'Oreal-Unesco Italy award 'For Women in Science' (2014), the International Society for Pediatric and Adolescent Diabetes (ISPAD) Young Investigator award (2015), the European Society for Paediatric Endocrinology Young Investigator award (2016), and the G.B. Morgagni Silver Medal (2016).

DOI: https://doi.org/10.17925/USE.2018.14.1.20

\section{Keywords}

Pediatric diabetes, insulin sensitivity, agespecific approach, insulin pump, continuous glucose monitoring, DPP-4 inhibitors, GLP1 receptor agonists, SGLT2 inhibitors

Disclosure: M Loredana Marcovecchio has nothing to disclose in relation to this article.

Review Process: This is an expert interview and as such has not undergone the journal's standard peer review process.

Authorship: All named authors meet the International Committee of Medical Journal Editors (ICMJE) criteria for authorship of this manuscript, take responsibility for the integrity of the work as a whole, and have given final approval to the version to be published.

open Access: This article is published under the Creative Commons Attribution Noncommercial License, which permits any noncommercial use, distribution, adaptation, and reproduction provided the original author(s) and source are given appropriate credit. (CThe Authors 2018

Received: April 22, 2018

Published Online: May, 22, 2018

Citation: US Endocrinology. 2018;14(1):20-21

Corresponding Author: M. Loredana Marcovecchio, University of Cambridge, Box 116, Level 8, Cambridge Biomedical Campus, Hills Road, Cambridge, CB2

OQQ, UK. E: mlm45@medschl.cam.ac.uk

Support: No funding was received in

the publication of this article.
$\mathrm{T}$ he needs of pediatric patients with diabetes differ from those of adults. Challenges include growth and development, and care outside of the home, such as in the school setting. In addition, in type 2 diabetes, there are fewer treatment options for pediatric patients; while recent years have seen the approval of many glucose-lowering drugs for adults, only metformin and insulin are approved in patients aged under 18 years. In an expert interview, M Loredana Marcovecchio, of the University of Cambridge, UK, discusses recent advances and remaining challenges in the treatment of pediatric patients with both type 1 and type 2 diabetes.

\section{Q. What are the major challenges of treating pediatric type 1 and 2 diabetes?}

Achieving optimal glycemic control in children and adolescents with diabetes remains a major challenge. Management of diabetes requires an age-specific approach, taking into account the needs and characteristics related to specific developmental stages. Variable eating patterns and physical activity, along with the limited ability to communicate symptoms of hypoglycemia/hyperglycemia are the main challenges in very young children. During adolescence, changes in insulin sensitivity related to physical growth and pubertal hormonal changes, psychological and behavioral issues and poor compliance can complicate diabetes management. Additional factors contributing to challenges in young people with diabetes include family dynamics, health status and care outside home, such as in the school setting.

\section{Q. What have been the most important advances in the treatment of type 1 diabetes in children?}

Insulin is the cornerstone of treatment of type 1 diabetes. Since its discovery almost a century ago, there have been key milestones which have improved treatment: the introduction of insulin analogues with better pharmacokinetic/pharmacodynamic profiles; the implementation of newer devices for insulin administrations; and alternative ways of insulin delivery. Of particular note are the recent advances in 
diabetes technology, these include insulin pump therapy, continuous glucose monitoring and sensor-augmented insulin pump therapy, which are leading the way towards the development of a 'closed-loop system' or 'artificial pancreas'.

Developments in diabetes technology are fast-moving and hold promise to improve quality of life and outcomes. However, technology does not necessarily 'fit' every patient, and in children and adolescents its introduction in daily routine care can pose some psychological and 'timeeffort' burdens, which need to be addressed.

\section{Q. How do you treat type 2 diabetes in children who cannot meet their glycemic targets using metformin and lifestyle intervention?}

Based on current recommendations, lifestyle interventions represent the first-line of managing pediatric type 2 diabetes. Metformin can be introduced if there is no benefit of lifestyle interventions.

Unfortunately, compared to adult-onset type 2 diabetes, there are fewer treatment options for youth. Up to now, metformin and insulin are the only agents approved for the management of type 2 diabetes in adolescents. Therefore, when glycemic targets are not reached, insulin is the only additional approved therapeutic option. A long-acting insulin can be firstly introduced and, if this is not enough, the addition of a meal-time short-acting insulin is the next step.

\section{Q. Is there any evidence to support the use of other antidiabetic drugs, such as dipeptidyl peptidase-4 inhibitors, glucagon-like peptide-1 receptor agonists, or sodium-glucose cotransporter 2 , in patients aged under 18 years?}

Whereas many antidiabetic drugs are licensed for use in adults with type 2 diabetes in the US and Europe, only metformin is approved for pediatric use in most countries. Many clinical trials of anti-hyperglycemic agents are underway in youth-onset type 2 diabetes (NCT03429543, NCT01554618, NCT03170518) should be available in a few years.

\section{Q. Of the above drugs, which would you expect to be most useful in a pediatric population?}

There is a strong need for future studies assessing each of the above drugs in pediatric type 2 diabetes, due to potential differences in safety and efficacy profiles between different age groups. In adults, drugs such as sodium-glucose cotransporter 2 (SLGT2) inhibitors have been associated not only with reductions in glycated hemoglobin (HbA1c), but with other beneficial effects on weight loss, blood pressure, renal function and cardiovascular outcomes. Given that type 2 diabetes often presents a more aggressive course in the pediatric population with high rates of complications already at the time of diagnosis, SLGT2 inhibitors might be a useful therapeutic option in this age group. 